\title{
NEAT1 Enhances MPP+-Induced Pyroptosis In SH- SY5Y Cells Via Targeting Mir-5047/YAF2 Signaling
}

\section{Hong Shen}

Second People's Hospital, Wujiang District

\section{Hui Song}

TaiHe Hospital, Hubei University of Medicine

\section{Songlin Wang}

TaiHe Hospital, Hubei University of Medicine

\section{Daojing Su}

TaiHe Hospital, Hubei University of Medicine

Qiang Sun ( $\square$ sunnybo0681@sina.com)

TaiHe Hospital, Hubei University of Medicine https://orcid.org/0000-0002-1050-5067

\section{Research Article}

Keywords: Parkinson's disease, nuclear paraspeckle assembly transcript 1, pyroptosis, inflammasomes

Posted Date: January 11th, 2022

DOI: https://doi.org/10.21203/rs.3.rs-1199805/v1

License: (a) (i) This work is licensed under a Creative Commons Attribution 4.0 International License. Read Full License 


\section{Abstract}

Parkinson's disease (PD) is the second most frequent neurodegenerative disease. The aim of our study is to explore the role and regulatory mechanism of long non-coding RNA (IncRNA) NEAT1 in the MPP ${ }^{+}$ induced neuron pyroptosis. The levels of miR-5047 and YAF2 mRNA were determined through qRT-PCR. TUNEL staining was carried out to analyze neuronal apoptosis. Luciferase activity assay was accomplished to analyze the combination of miR-5047 and NEAT1 and YAF2 3'-UTR. Besides, the concentrations of IL-1 $\beta$ and IL-18 in supernatant were analyzed by ELISA assay. The levels of protein were examined through Western blot. NEAT1 and YAF2 expression were increased, while miR-5047 level was declined in the SH-SY5Y cells treated with MPP ${ }^{+}$. NEAT1 was a positively regulator for the SH-SY5Y cells pyroptosis induced by MPP ${ }^{+}$. In addition, YAF2 was a downstream target of miR-5047. NEAT1 promoted YAF2 expression via inhibiting miR-5047. Importantly, the promotion of NEAT1 to SH-SY5Y cells pyroptosis induced by $\mathrm{MPP}^{+}$was recused by miR-5047 mimic transfection and YAF2 downregulation. In conclusion, NEAT1 was increased in the SH-SY5Y cells treated with MPP ${ }^{+}$, and it promoted the $\mathrm{MPP}^{+}$-induced pyroptosis through facilitating YAF2 expression by sponging miR-5047.

\section{Introduction}

Over the last few decades, central nervous system diseases have become a major cause of disability worldwide. Common central nervous system diseases include Alzheimer's disease, metastatic brain tumor, stroke and Parkinson's disease (PD) (Morofuji \& Nakagawa, 2020). Among them, PD is the second most frequent neurodegenerative disease, and is characterized by the non-motor symptoms like cognitive impairment and gastrointestinal dysfunction, and the motor symptoms like bradykinesia, postural instability and resting tremor (Calvani \& Picca, 2020,Camacho \& Greenland, 2021,Filippi et al., 2020). Ageing, genetic, and environment risk factors or other pathogenic factors lead to the loss of neuron in several brain areas, especially in substantia nigra. The mutations of $L R R K 2$, PRKN, PINK1, ATP13A2, and DJ-1 are several known pathogenic genes for PD (McDonald et al., 2018,Zhao et al., 2020). Currently, although a great advance in the technology used in drug discovery has been made, the development of the drugs used in PD treatment remains challenging, which are due to the complex pathogenesis of the disease.

Numerous lines of evidence indicate that chromic neuroinflammation is one of the mainly hallmarks of PD, while whether inflammation increasing is a consequence or cause of PD remains unclear (Belarbi et al., 2020,Pajares et al., 2020). Pyroptosis has been first introduced by Cookson et al. in 2001, which is mediated by caspase- 1 and accompanied by the release of a series cytokines, such as interleukin (IL)- $1 \beta$ and IL-18 (Liu et al., 2021). During pyroptosis, the activation of caspase-1 can be induced by inflammasome, such as NLR family pyrin domain-containing (NLRP) 3 or NLRP1 inflammasome (Lu et al., 2020,Tsuchiya, 2020). It was reported that the expression of NLRP3 inflammasome- and pyroptosisrelated proteins was highly expressed and pyroptosis was activated in the 1-methyl-4-phenylpyridinium $\left(\mathrm{MPP}^{+}\right)$-treated SH-SY5Y cells (Zeng et al., 2019). Inhibition of NLRP3 inflammasome-mediated 
pyroptosis in MPTP-induced PD mice model and in MPP -treated PC12 cells could effectively attenuate the neuron apoptosis (Zhang et al., 2020). Currently, the major inflammasome type studied in the pathogenesis of PD is mediated by NLRP3, but there are few studies on the role of NLRP1 inflammasome in the development of PD. Chen et al. demonstrated that the expression of NLRP1 is decreased in the antiinflammatory drugs-treated a-synuclein-stimulated BV-2 cells (Chen et al., 2021).

The thorough annotation of the human genome by the GENCODE and ENCODE projects indicated that many transcription products of the human DNA are long non-coding RNAs (IncRNAs), and more than ten thousand IncRNAs have been identified (Martens-Uzunova et al., 2014,St Laurent et al., 2015). A number of IncRNA is known to be involved in the regulation of PD. For instance, Simchovitz et al. provided a new IncRNA LINC-PINT that is increased in the substantia nigra of PD patients and other PD models may play a neuroprotective role in PD (Simchovitz et al., 2020). Among the multiple IncRNAs, the role of nuclear paraspeckle assembly transcript 1 (NEAT1) in PD is contradictory, that has attracted particular interest. In our previous study, the results demonstrated that the expression of NEAT1 is highly expressed in the SHSY5Y cells treated with MPP ${ }^{+}$, and that silencing of NEAT1 obviously attenuate the neuronal apoptosis induced by MPP ${ }^{+}$(Sun et al., 2021b). In addition, some studies reported that NEAT1 induces the activation of inflammasome and its-mediated pyroptosis in different diseases (Zhan et al., 2020,Zhang et al., 2019). In this study, we probed the functions of NEAT1 in the SH-SY5Y cells pyroptosis induced by $\mathrm{MPP}^{+}$, and the regulatory mechanism of NEAT1 on pyroptosis. Our work proved a new evidence for the potential of NEAT1 in PD treatment.

\section{Materials And Methods}

\section{Cell culture and treatment}

In our study, SH-SY5Y cells were obtained from the ATCC (Manassas, Va., USA). MPP ${ }^{+}$was got from the Sigma Company (St. Louis, MO, USA), and was utilized to induce disease model at the dosage of $100 \mu \mathrm{M}$ for 24 hours. The complete Dulbecco's Modified Eagle medium (DMEM) medium used in our study is composed of basal DMEM medium (Gibco, Grand Island, NY, USA), 10\% fetal bovine serum (Gibco), as well as $1 \%$ penicillin/streptomycin (Gibco). Here, the treated and un-treated $\mathrm{SH}-\mathrm{SY} 5 \mathrm{Y}$ cells were cultured in the complete DMEM medium. The NEAT1 siRNA, siRNA negative control, miR-5047 mimics, mimics negative control, miR-5047 inhibitor, inhibitor negative control, and YY1-associated factor 2 (YAF2) siRNA were synthesized by GenScript Company (Nanjing, China). All above gene segments, the NEAT1 overexpression plasmid, as well as empty plasmid were transfected into SH-SY5Y cells using Lipofectamine 3000 reagent (Invitrogen, Carlsbad, CA).

\section{Detection Of Proteins Expression}

The expression levels of pro-caspase-1, cleaved caspase-1, YAF2, NLRP1 and adaptor apoptosisassociated speck-like (ASC) were determined using Western blot. Collected SH-SY5Y cells were washed 
with 0.01 M PBS solution for twice. Then, the cells were broken using RIPA lysis solution (Solarbio, Beijing, China) for protein extraction. Next, $20 \mu \mathrm{g}$ protein for each group were separated by $12 \%$ SDSPAGE gels for $2.5 \mathrm{~h}$, and were transferred into polyvinylidene fluoride membranes. After that, membranes were blocked with $5 \%$ fresh non-fat milk for 1 hour followed by were maintained with the antibodies overnight at $4^{\circ} \mathrm{C}$. The pro-caspase- 1 , anti-ASC, anti-NLRP1, anti- anti-cleaved caspase- 1 , and anti- $\beta$-actin (internal reference) were purchased from Abcam (Cambridge, MA). All primary antibodies were diluted at a ratio of 1:2000 when we used. Next day, membranes were maintained with secondary antibodies for 1 hour. Finally, an ECL kit was utilized to analyze the protein bands.

\section{Detection Of Theneat1, Mir-5047 And Yaf2 Mrna Levels}

The levels of NEAT1, miR-5047, and YAF2 mRNA were measured using qRT-PCR assay. SH-SY5Y cells were collected, and were washed with PBS for twice. Then, the total RNA was isolated using TRIzol reagent (Invitrogen), and was reversed transcription into complementary DNA usage the First-Strand Synthesis Kit (Invitrogen). Next step, the qPCR experiments were accomplished using SYBR Green PCR Master Mix Kit (Invitrogen). All steps were carried out strictly according to the instructions. In this study, the expression of NEAT1 and YAF2 mRNA normalized to GAPDH, and U6 served as the internal reference for miR-5047. The levels of NEAT1, miR-5047, and YAF2 mRNA were calculated through $-2^{\triangle \Delta C t}$ method.

\section{Determination Of The Apoptotic Rate In Sh-sy5y Cells}

The apoptotic rate of SH-SY5Y cells was measured using TUNEL staining. SH-SY5Y cells at a density of 5 $.10^{4}$ cells/well were inoculated into 24-well plates, and were treated with $\mathrm{MPP}^{+}$or transfected for 24 hours. After that, the cells were fixed with $4 \%$ formaldehyde fixative buffer (Solarbio) for 1 hour, and were washed with PBS solution. After incubation with $3 \% \mathrm{H}_{2} \mathrm{O}_{2}$ solution for $15 \mathrm{~min}$, the treated and untreated SH-SY5Y cells were maintained with $0.1 \%$ Triton X-100 in PBS solution for 10 min. Subsequently, the apoptotic cells were marked by TUNEL solution (Solarbio) in accordance with the instruction. After incubation with DAPI solution in the dark for 5 min to mark nucleuses, the number of apoptotic cells (TUNEL-positive cells) were counted using Image $\mathrm{J}$ software.

\section{Detection of the interaction of miR-5047 with NEAT1 and YAF2 3'-UTR}

Dual-luciferase activity assay was fulfilled to determine the combination of NEAT1 and miR-5047, and the combination of miR-5047 and YAF2 3'-UTR. Here, the wild-type (WT) and mutated (Mut) gene sequences of NEAT1 and YAF2 3'-UTR containing the binding sites with miR-5047 were sub-cloned into the luciferase reporter plasmid. The recombinant plasmids of NEAT1-WT, NEAT1-Mut, YAF2 3'-UTR-WT, and YAF2 3'UTR-Mut were transfected with miR-5047 mimics or mimics negative control into 293T cells. The luciferase activity was determined usage Dual-Luciferase Reporter Assay System (Promega, WI, USA). 


\section{Detection Of The II-1 $\beta$ And II-18 Levels In Supernatant}

The levels of IL-1 $\beta$ and IL-18 in the supernatant of SH-SY5Y cells were measured through ELISA assay according to the protocols of human IL-1 $\beta$ ELISA kit and human IL-18 ELISA kit (R\&D Systems).

\section{Statistical analysis}

The results in our study were analyzed using the SPSS 20.0 software (IBM., Armonk, NY), and the images were marked through GraphPad Prism 6.0. The data were presented as mean \pm SD. All experiments were repeated for three time at least, and the value of $\mathrm{P}<0.05$ was recognized statistically significant.

Student's $t$-test and ANOVA analysis were utilized for the analysis between two groups and among multiple groups.

\section{Results}

NEAT1 and YAF2 were increased, pyroptosis was activated, and miR-5047 was decreased in the MPP+induced SH-SY5Y cells

To investigate the expression of NEAT1, miR-5047 and YAF2 in PD, we induced PD model in vitro using SH-SY5Y cells. The levels of NEAT1 and YAF2 mRNA were increased, while miR-5047 was decreased in the SH-SY5Y cells treated with $\mathrm{MPP}^{+}$(Figure 1A). The western blotting assay results also indicated that YAF2 was increased in the SH-SY5Y cells treated with $\mathrm{MPP}^{+}$(Figure 1B). Meanwhile, we also detected the level of NLRP1 inflammasomes-mediated pyroptosis in MPP'-induced SH-SY5Y cells. As shown in figure 1C, the expression of cleaved caspase-1, pro-caspase-1, NLRP1, and ASC was highly expressed in the SHSY5Y cells treated with MPP ${ }^{+}$. The levels of IL-1 $\beta$ and IL-18 were higher in the supernatant of MPP ${ }^{+}$ treated SH-SY5Y cells than that in the untreated cells (Figure 1D). Overall, the expression of NEAT1 and YAF2 was increased, NLRP1 inflammasome-mediated pyroptosis was activated, while miR-5047 expression was declined in the SH-SY5Y cells treated with MPP ${ }^{+}$.

\section{NEAT1 increasing aggravated the SH-SY5Y cells pyroptosis induced by MPP+}

To probe the effect of NEAT1 on the pyroptosis induced by MPP ${ }^{+}$in SH-SY5Y cells, we constructed the NEAT1 overexpression plasmid and synthetized the NEAT1 siRNA. The expression of NEAT1 was significantly upregulated by the transfection of NEAT1 overexpression plasmid, and was downregulated by the transfection of NEAT1 siRNA (Figure 2A). NEAT1 increasing could enhance the cells apoptosis, while NEAT1 decreasing attenuated the cell apoptosis in the SH-SY5Y cells treated with MPP ${ }^{+}$(Figure 2B and $2 \mathrm{C}$ ). In the SH-SY5Y cells treated with MPP ${ }^{+}$, NEAT1 increasing facilitated pro-caspase-1, NLRP1, ASC, and cleaved caspase-1 expression, but NEAT1 decreasing suppressed the expression of above factors (Figure 2D). Consistently, NEAT1 increasing promoted the secretion of IL-1 $\beta$ and IL-18, and NEAT1 silencing reduced the secretion of IL-1 $\beta$ and IL-18 in the SH-SY5Y cells treated with MPP ${ }^{+}$(Figure $2 \mathrm{E}$ and 2F). In conclusion, NEAT1 promoted the pyroptosis in the SH-SY5Y cells treated with MPP . 


\section{Neat1 Promoted Yaf2 Expression Via Inhibiting Mir-5047}

Furthermore, our data certificated that NEAT1 increasing declined miR-5047 level, and NEAT1 decreasing facilitated miR-5047 expression in the SH-SY5Y cells treated with MPP $^{+}$(Figure 3A). NEAT1 increasing boosted YAF2 mRNA and protein expression, and NEAT1 silencing restrained YAF2 mRNA and protein expression in the SH-SY5Y cells treated with $\mathrm{MPP}^{+}$(Figure 3B and $3 \mathrm{C}$ ). The mechanism of competitive endogenous RNAs (ceRNAs) proposes that IncRNAs compete miRNA with mRNA, thus to reduce the inhibition of miRNAs to mRNA (Li et al., 2020). Here, we predicted the binding sites between NEAT1 and miR-5047 using starBase v2.0 database (Figure 3D), as well as the binding sites between miR-5047 and YAF2 3'-UTR using TargetScan Human database (Figure 3E). We proved the combination of NEAT1 and miR-5047 usage luciferase reporter assay (Figure 3F), and also confirmed that YAF2 3'-UTR was a downstream target of miR-5047 (Figure 3G). To probe whether NEAT1 affects the expression of YAF2 through miR-5047, the miR-5047 mimics was co-transfected with NEAT1 overexpression plasmid into the SH-SY5Y cells treated with MPP ${ }^{+}$. NEAT1 increasing promoted the YAF2 mRNA expression, which was reversed by miR-5047 mimics transfection (Figure 3H). NEAT1 increasing-induced YAF2 protein expression also was partly rescued by miR-5047 increasing (Figure 3I). In addition, the inhibition of NEAT1 decreasing to YAF2 mRNA and protein expression was reversed by miR-5047 decreasing (Figure $3 \mathrm{~J}$ and $3 \mathrm{~K}$ ). In a word, NEAT1 promoted the expression of YAF2 through sponging miR-5047.

\section{Increasing miR-5047 partly reversed the promotion of NEAT1 to MPP+-induced pyroptosis}

Based on above results, we asked that whether NEAT1 enhances the pyroptosis in the SH-SY5Y cells treated with $\mathrm{MPP}^{+}$through miR-5047/YAF2 signaling pathway. NEAT1 overexpression plasmid was cotransfected with miR-5047 mimics into the SH-SY5Y cells treated with MPP ${ }^{+}$. Our results certificated that the promotion of NEAT1 to the SH-SY5Y cells apoptosis induced by $\mathrm{MPP}^{+}$was reversed by miR-5047 increasing (Figure 4A and 4B). The promotion of NEAT1 to pro-caspase-1, NLRP1, ASC, and cleaved caspase- 1 expression also was recused by miR-5047 increasing (Figure 4C). In addition, miR-5047 overexpression recused the promotion of NEAT1 to IL- $\beta$ and IL-18 secretion (Supplementary figure 1A and 1B). The promotion of NEAT1 to SH-SY5Y cells pyroptosis induced by $\mathrm{MPP}^{+}$was recused by miR-5047 overexpression.

\section{YAF2 silencing partly reversed the promotion of NEAT1 to MPP+-induced pyroptosis}

We also co-transfected the NEAT1 overexpression plasmid with YAF2 siRNA into the SH-SY5Y cells treated with $\mathrm{MPP}^{+}$. The results indicated that the promotion of NEAT1 to SH-SY5Y cells apoptosis induced by $\mathrm{MPP}^{+}$was reversed following YAF2 downregulation (Figure 5A and 5B). Consistently, the upregulation of pro-caspase-1, NLRP1, ASC, and cleaved caspase-1 induced by NEAT1 was also suppressed by YAF2 decreasing (Figure 5C). The promotion of NEAT1 to IL-1 $\beta$ and IL-18 secretion also was recused by YAF2 decreasing (Supplementary figure 2A and 2B). In short, the promotion of NEAT1 to the SH-SY5Y cells pyroptosis induced by MPP ${ }^{+}$was recused by YAF2 decreasing. 


\section{Discussion}

In our present study, the data demonstrated that NEAT1 is highly expressed in the SH-SY5Y cells treated with $\mathrm{MPP}^{+}$, and it enhances the MPP+-induced pyroptosis through promoting YAF2 expression by sponging miR-5047. Here, we explored the role of NEAT1 in the neuron pyroptosis induced by MPP ${ }^{+}$, and the regulatory mechanism of it in the pyroptosis.

A growing body of studies indicated that IncRNAs are promising target for PD treatment. Yang et al. reported that IncRNA HOXA-AS2 is increased in the peripheral blood mononuclear cells of the patients with PD, and it maybe participate in the development of PD through regulating microglial-mediated neuroinflammation via interacting with the polycomb repressive complex 2 (Yang et al., 2021). Another IncRNA, lincRNA-p21, could exacerbate the microglial-mediated neuroinflammation and the loss of $\mathrm{TH}^{+}$ neuron in the PD mouse model induced by MPTP (Ye et al., 2018). MPTP/MPP ${ }^{+}$is a typical drug, which has been used for the establishment of PD model. Here, our data showed that the level of IncRNA NEAT1 is increased in the SH-SY5Y cells treated with MPP'. NEAT1 is a well-studied IncRNA in multiply disorders. It was reported that NEAT1 is involved in a series physiological and pathological processes, including cancer, immune response, neurodegenerative disorders, and viral infection (Wang et al., 2020). The role and action mechanism of NEAT1 in inflammation-induced neuron apoptosis in PD, however, has not yet been fully clarified.

Neuroinflammation is one of the major cause of the loss of $\mathrm{TH}^{+}$neuron in PD, which can be induced by activated inflammasome-mediated pyroptosis. Inflammasome is critical mediator of the innate immune response to infection. Currently, NLRP1, NLRP3, NLRC4, IPAF, and absent in melanoma 2-mediated inflammasomes are the most well-known inflammasomes (Ravichandran \& Heneka, 2021). NLRP1 inflammasome consist of NLRP1, ASC, and pro-caspase-1. Inflammasomes induces the activation of caspase-1, and then activated caspase-1 cleaves gasdermin $D$, and promotes the release of IL-1 $\beta$ and IL18 (Lünemann \& Malhotra, 2021). In this study, our data revealed that the MPP ${ }^{+}$-induced activation of NLRP1 inflammasome-mediated pyroptosis can be enhanced by NEAT1 overexpression, and attenuated by NEAT1 downregulation. To explore the regulatory mechanism of NEAT1 to neuron pyroptosis, we predicated the binding sites between NEAT1 and miR-5047. Our results showed that the level of miR-5047 is decreased in the SH-SY5Y cells treated with MPP ${ }^{+}$. The combination of NEAT1 and miR-5047 was proved usage luciferase reporter assay. Meanwhile, our data indicated that the promotory effect of NEAT1 on the SH-SY5Y cells pyroptosis induced by MPP ${ }^{+}$is recused followed by miR-5047 overexpression.

Transcription factor Yin-Yang-1 (YY1) is a crucial regulator for gene expression, and participates in the regulation of many disorders including PD (Tiwari \& Pal, 2017). It was reported that YY1 could regulate neuroinflammation development through suppressor of cytokine signaling 3 (Sun et al., 2021a). YAF2 was first identified in a yeast two-hybrid screen for the transcriptional factor of $Y Y 1$, and was proved to be interacted with YY1 in muscle differentiation and development (Kalenik et al., 1997,Zhuang et al., 2019). At present, the function of YAF2 in PD remains unreported. In this present, YAF2 is downregulated in the 
SH-SY5Y cells treated with MPP ${ }^{+}$, and is a target of miR-5047. NEAT1 could promote YAF2 expression by acting as miR-5047 sponge. Importantly, the promotory effect of NEAT1 on the SH-SY5Y cells pyroptosis induced by $\mathrm{MPP}^{+}$is partly recused by YAF2 downregulation. YAF2 maybe mediate the regulatory of NEAT1 to the pyroptosis in SH-SY5Y cells induced by MPP ${ }^{+}$.

\section{Conclusion}

In conclusion, our data demonstrated that NEAT1 could aggravate MPP ${ }^{+}$-induced pyroptosis in SH-SY5Y cells through promoting YAF2 by sponging miR-5047. Our data indicated that NEAT1 may be a promising target for the treatment of PD.

\section{Statements And Declarations}

\section{Funding}

The authors declare that no funds, grants, or other support were received during the preparation of this manuscript.

\section{Competing Interests}

The authors have no relevant financial or non-financial interests to disclose.

\section{Author Contribution}

Hong Shen, Hui Song and Qiang Sun contributed to the conception of the study and helped perform the analysis with constructive discussion. Hong Shen, Hui Song, Songlin Wang, and Daojing Su performed the experiment; contributed significantly to analysis and manuscript preparation; performed the data analyses. Hong Shen and Qiang Sun contributed to write the manuscript.

\section{Ethics Approval and Consent to Participate}

This is a cellular study. The TaiHe Hospital Research Ethics Committee has confirmed that no ethical approval is required.

\section{Consent to participate}

Not applicable.

\section{Consent to publish}

Not applicable.

\section{Data Availability}


The datasets generated during and/or analyzed during the current study are available from the corresponding author on reasonable request.

\section{Availability of data and materials}

Not applicable.

\section{References}

1. Belarbi K, Cuvelier E, Bonte MA, Desplanque M, Gressier B, Devos D, Chartier-Harlin MC (2020) Glycosphingolipids and neuroinflammation in Parkinson's disease. Mol Neurodegener 15 (1): 59.

2. Calvani R, Picca A (2020) A novel multi-marker discovery approach identifies new serum biomarkers for Parkinson's disease in older people: an EXosomes in PArkiNson Disease (EXPAND) ancillary study. Geroscience 42 (5): 1323-1334.

3. Camacho M, Greenland JC (2021) The Gastrointestinal Dysfunction Scale for Parkinson's Disease. Mov Disord 36 (10): 2358-2366.

4. Chen CM, Yen CY, Chen WL, Lin CH, Wu YR, Chang KH, Lee-Chen GJ (2021) Pathomechanism Characterization and Potential Therapeutics Identification for Parkinson's Disease Targeting Neuroinflammation. Int J Mol Sci 22 (3): 1062.

5. Filippi M, Canu E, Donzuso G, Stojkovic T, Basaia S, Stankovic I, Tomic A, Markovic V, Petrovic I, Stefanova E, Kostic VS, Agosta F (2020) Tracking Cortical Changes Throughout Cognitive Decline in Parkinson's Disease. Mov Disord 35 (11): 1987-1998.

6. Kalenik JL, Chen D, Bradley ME, Chen SJ, Lee TC (1997) Yeast two-hybrid cloning of a novel zinc finger protein that interacts with the multifunctional transcription factor YY1. Nucleic Acids Res 25 (4): 843-849.

7. Lünemann JD, Malhotra S (2021) Targeting Inflammasomes to Treat Neurological Diseases. Ann Neurol 90 (2): 177-188.

8. Li W, Yu W, Jiang X, Gao X, Wang G, Jin X, Zhao Z, Liu Y (2020) The Construction and Comprehensive Prognostic Analysis of the LncRNA-Associated Competitive Endogenous RNAs Network in Colorectal Cancer. Front Genet 11: 583.

9. Liu P, Zhang Z, Li Y (2021) Relevance of the Pyroptosis-Related Inflammasome Pathway in the Pathogenesis of Diabetic Kidney Disease. Front Immunol 12: 603416.

10. Lu F, Lan Z, Xin Z, He C, Guo Z, Xia X, Hu T (2020) Emerging insights into molecular mechanisms underlying pyroptosis and functions of inflammasomes in diseases. J Cell Physiol 235 (4): 32073221.

11. Martens-Uzunova ES, Böttcher R, Croce CM, Jenster G, Visakorpi T, Calin GA (2014) Long noncoding RNA in prostate, bladder, and kidney cancer. Eur Urol 65 (6): 1140-1151.

12. McDonald C, Gordon G, Hand A, Walker RW, Fisher JM (2018) 200 Years of Parkinson's disease: what have we learnt from James Parkinson? Age Ageing 47 (2): 209-214. 
13. Morofuji Y, Nakagawa S (2020) Drug Development for Central Nervous System Diseases Using In vitro Blood-brain Barrier Models and Drug Repositioning. Curr Pharm Des 26 (13): 1466-1485.

14. Pajares M, A IR, Manda G, Boscá L, Cuadrado A (2020) Inflammation in Parkinson's Disease: Mechanisms and Therapeutic Implications. Cells 9 (7): 1687.

15. Ravichandran KA, Heneka MT (2021) Inflammasome activation in neurodegenerative diseases. Essays Biochem EBC20210021.

16. Simchovitz A, Hanan M, Yayon N, Lee S, Bennett ER, Greenberg DS, Kadener S, Soreq H (2020) A IncRNA survey finds increases in neuroprotective LINC-PINT in Parkinson's disease substantia nigra. Aging Cell 19 (3): e13115.

17. St Laurent G, Wahlestedt C, Kapranov P (2015) The Landscape of long noncoding RNA classification. Trends Genet 31 (5): 239-251.

18. Sun M, Sun Y, Ma J, Li K (2021a) YY1 promotes SOCS3 expression to inhibit STAT3-mediated neuroinflammation and neuropathic pain. Mol Med Rep 23 (2): 103.

19. Sun Q, Zhang Y, Wang S, Yang F, Cai H, Xing Y, Chen Z, Chen J (2021b) NEAT1 Decreasing Suppresses Parkinson's Disease Progression via Acting as miR-1301-3p Sponge. J Mol Neurosci 71 (2): 369-378.

20. Tiwari PC, Pal R (2017) The potential role of neuroinflammation and transcription factors in Parkinson disease. Dialogues Clin Neurosci 19 (1): 71-80.

21. Tsuchiya K (2020) Inflammasome-associated cell death: Pyroptosis, apoptosis, and physiological implications. Microbiol Immunol 64 (4): 252-269.

22. Wang Z, Li K, Huang W (2020) Long non-coding RNA NEAT1-centric gene regulation. Cell Mol Life Sci 77 (19): 3769-3779.

23. Yang X, Zhang Y, Chen Y, He X, Qian Y, Xu S, Gao C, Mo C, Chen S, Xiao Q (2021) LncRNA HOXA-AS2 regulates microglial polarization via recruitment of PRC2 and epigenetic modification of PGC-1a expression. J Neuroinflammation 18 (1): 197.

24. Ye Y, He X, Lu F, Mao H, Zhu Z, Yao L, Luo W, Sun X, Wang B, Qian C, Zhang Y, Lu G, Zhang S (2018) A lincRNA-p21/miR-181 family feedback loop regulates microglial activation during systemic LPS- and MPTP-induced neuroinflammation. Cell Death Dis 9 (8): 803.

25. Zeng R, Luo DX, Li HP, Zhang QS, Lei SS, Chen JH (2019) MicroRNA-135b alleviates MPP(+)mediated Parkinson's disease in in vitro model through suppressing Fox01-induced NLRP3 inflammasome and pyroptosis. J Clin Neurosci 65 125-133.

26. Zhan JF, Huang HW, Huang C, Hu LL, Xu WW (2020) Long Non-Coding RNA NEAT1 Regulates Pyroptosis in Diabetic Nephropathy via Mediating the miR-34c/NLRP3 Axis. Kidney Blood Press Res 45 (4): 589-602.

27. Zhang P, Cao L, Zhou R (2019) The IncRNA Neat1 promotes activation of inflammasomes in macrophages. Nat Commun 10 (1): 1495. 
28. Zhang X, Zhang Y, Li R, Zhu L, Fu B, Yan T (2020) Salidroside ameliorates Parkinson's disease by inhibiting NLRP3-dependent pyroptosis. Aging (Albany NY) 12 (10): 9405-9426.

29. Zhao Y, Qin L, Pan H, Liu Z, Jiang L, He Y, Zeng Q, Zhou X, Zhou X, Zhou Y, Fang Z, Wang Z, Xiang Y, Yang H, Wang Y, Zhang K, Zhang R, He R, Zhou X, Zhou Z, Yang N, Liang D, Chen J, Zhang X, Zhou Y, Liu H, Deng P, Xu K, Xu K, Zhou C, Zhong J, Xu Q, Sun Q, Li B, Zhao G, Wang T, Chen L, Shang H, Liu W, Chan P, Xue Z, Wang Q, Guo L, Wang X, Xu C, Zhang Z, Chen T, Lei L, Zhang H, Wang C, Tan J, Yan X, Shen L, Jiang H, Zhang Z, Hu Z, Xia K, Yue Z, Li J, Guo J, Tang B (2020) The role of genetics in Parkinson's disease: a large cohort study in Chinese mainland population. Brain 143 (7): 2220-2234.

30. Zhuang XF, Zhao LX, Guo SP, Wei S, Zhai JF, Zhou QH (2019) miR-34b inhibits the migration/invasion and promotes apoptosis of non-small-cell lung cancer cells by YAF2. Eur Rev Med Pharmacol Sci 23 (5): 2038-2046.

\section{Figures}

A

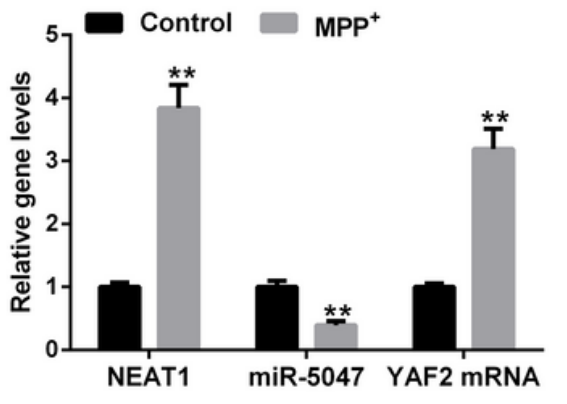

B

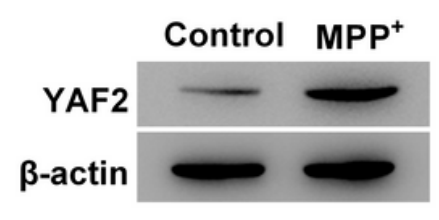

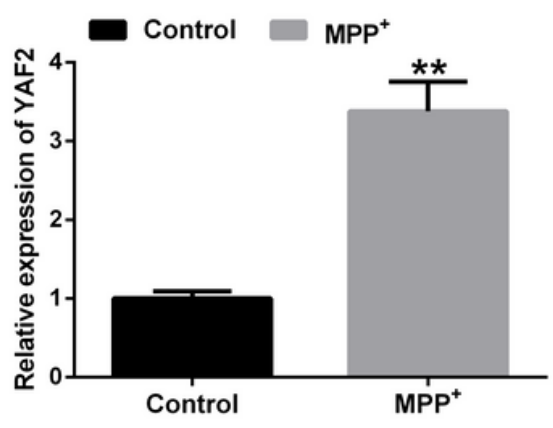

D

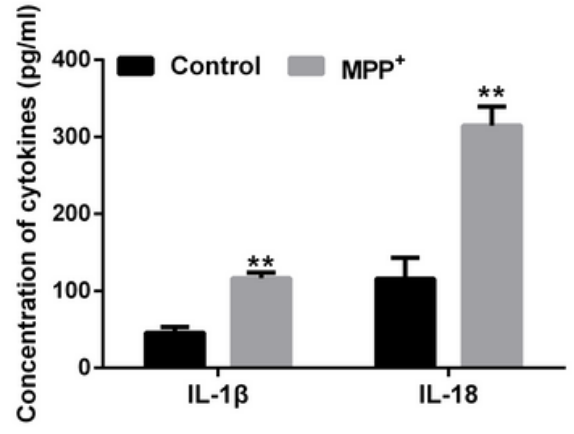

\section{Figure 1}

Detection of the levels of NEAT1, miR-5047, YAF2 mRNA and protein, and NLRP1 inflammasomes-related proteins. MPP ${ }^{+}$was used to establish PD model in vitro. (A) The expression levels of NEAT1, miR-5047, and YAF2 mRNA were determined usage qRT-PCR assay. (B) YAF2 protein level in MPP'-treated and untreated SH-SY5Y cells was measured using Western blot. (C) The levels of NLRP1 inflammasomes- 
mediated pyroptosis-related proteins (NLRP1, ASC, pro-caspase-1, and cleaved caspase-1) were analyzed also using Western blot. (D) The concentrations of IL-1 $\beta$ and IL-18 in the supernatant of SH-SY5Y cells were analyzed through ELISA assay. ${ }^{* \star} P<0.01$ vs. Control group.
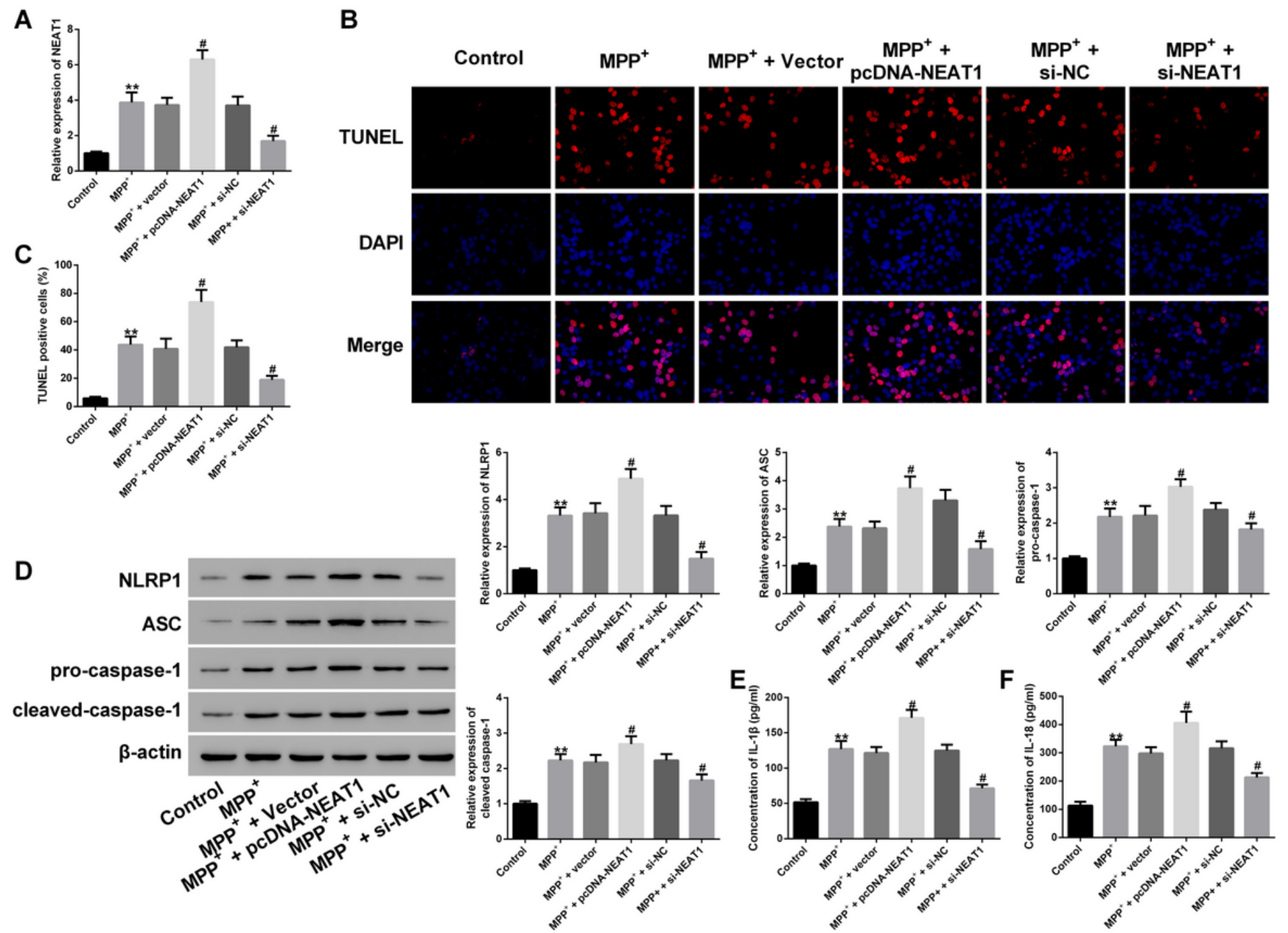

Figure 2

The effect of NEAT1 on MPP ${ }^{+}$-induced cell pyroptosis. Overexpression of NEAT1 and silencing the expression of NEAT1 in the MPP ${ }^{+}$-induced SH-SY5Y cells. (A) The expression level of NEAT1 was measured through qRT-PCR assay. (B and C) TUNEL staining was carried out to determine the apoptotic rate of SH-SY5Y cells. (D) The expression levels of NLRP1 inflammasomes-mediated pyroptosis were checked using Western blot. ( $E$ and $F$ ) The concentrations of IL-1 $\beta$ and IL-18 in the supernatant were determined by ELISA assay. ${ }^{* \star} P<0.01$ vs. Control group, ${ }^{\#} P<0.05$ vs. MPP+ group. 
A

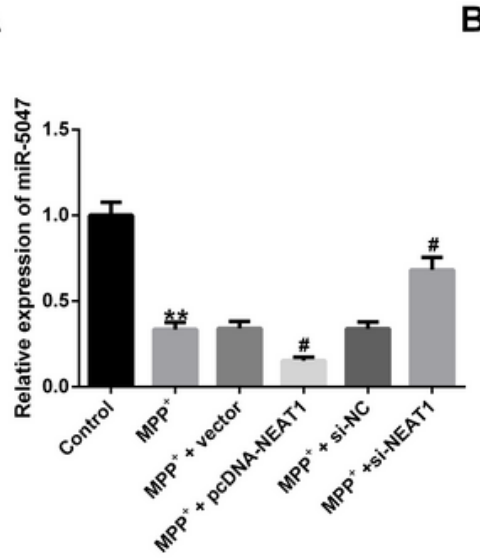

D

NEAT1-WT 5'-UCCUUCCCA - UG - AGCUGCAG-3 miR-5047 3'-UGGAAUGUUGGCGUCGACGUU-5'

NEAT1-MUT 5'-UCGAACGCU - UC - UCGAGCUG-3'
B

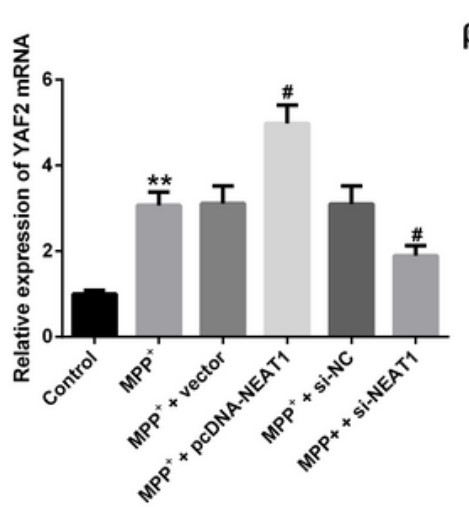

E

YAF2 mRNA-WT miR-5047

YAF2 mRNA-MUT
C YAF2 $--\cdots-\cdots$

$\beta$-actin --

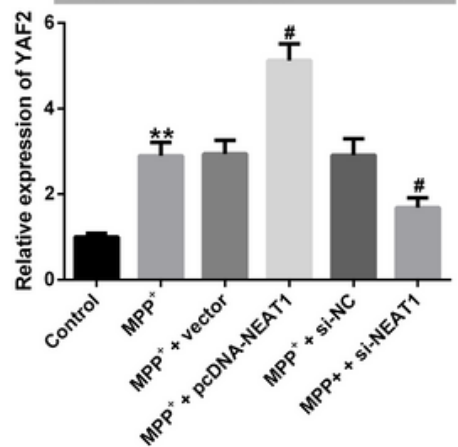

F

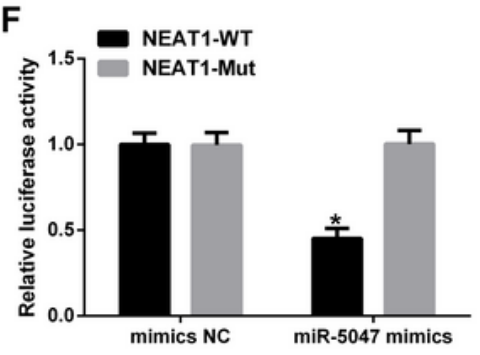

G

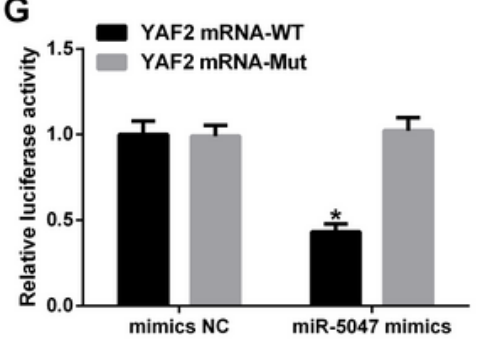

H
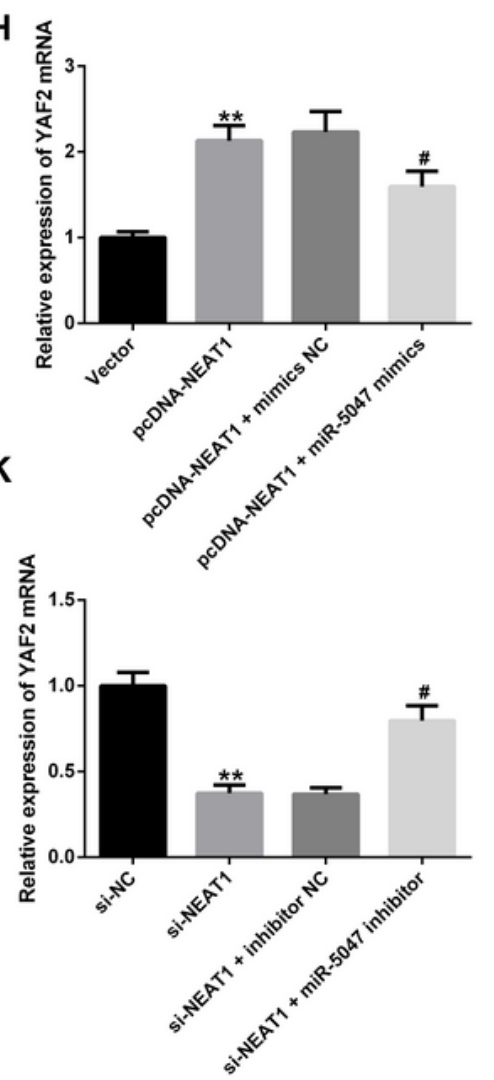

\section{Figure 3}

The combination of miR-5047 and NEAT1 and YAF2 3'-UTR. Overexpression of NEAT1 and silencing the expression of NEAT1 in the MPP ${ }^{+}$-induced SH-SY5Y cells. (A) The expression of miR-5047 was analyzed using qRT-PCR. (B) The expression of YAF2 mRNA was detected by qRT-PCR. (C) Western blot was performed to measure the expression of YAF2. ${ }^{*} P<0.01 \mathrm{vs}$. Control group, ${ }^{\#} P<0.05 \mathrm{vs}$. MPP+ group. (D) The binding sites between NEAT1 and miR-5047 was predicted by starBase v2.0 database. (E) The 
binding sites between miR-5047 and YAF2 3'-UTR was predicted using TargetScan Human database. (F and G) The interaction of miR-5047 and NEAT1 and YAF2 mRNA was determined through luciferase activity assay. NEAT1 overexpression plasmids were co-transfected with miR-5047 mimics into SH-SY5Y cells. ( $\mathrm{H}$ and I) qRT-PCR and Western blot were performed to determine the expression levels of YAF2 mRNA and protein, respectively. ${ }^{*} P<0.01 \mathrm{vs}$. Vector group, ${ }^{\#} P<0.05 \mathrm{vs}$. pcDNA-NEAT1 + mimics NC group. NEAT1 siRNAs were co-transfected with miR-5047 inhibitor into SH-SY5Y cells. ( $\mathrm{J}$ and K) qRT-PCR and Western blot were performed to determine the expression levels of YAF2 mRNA and protein, respectively. ${ }^{*} P<0.01$ vs. si-NC group, ${ }^{\#} P<0.05$ vs. si-NEAT1 + miR-5047 inhibitor group.

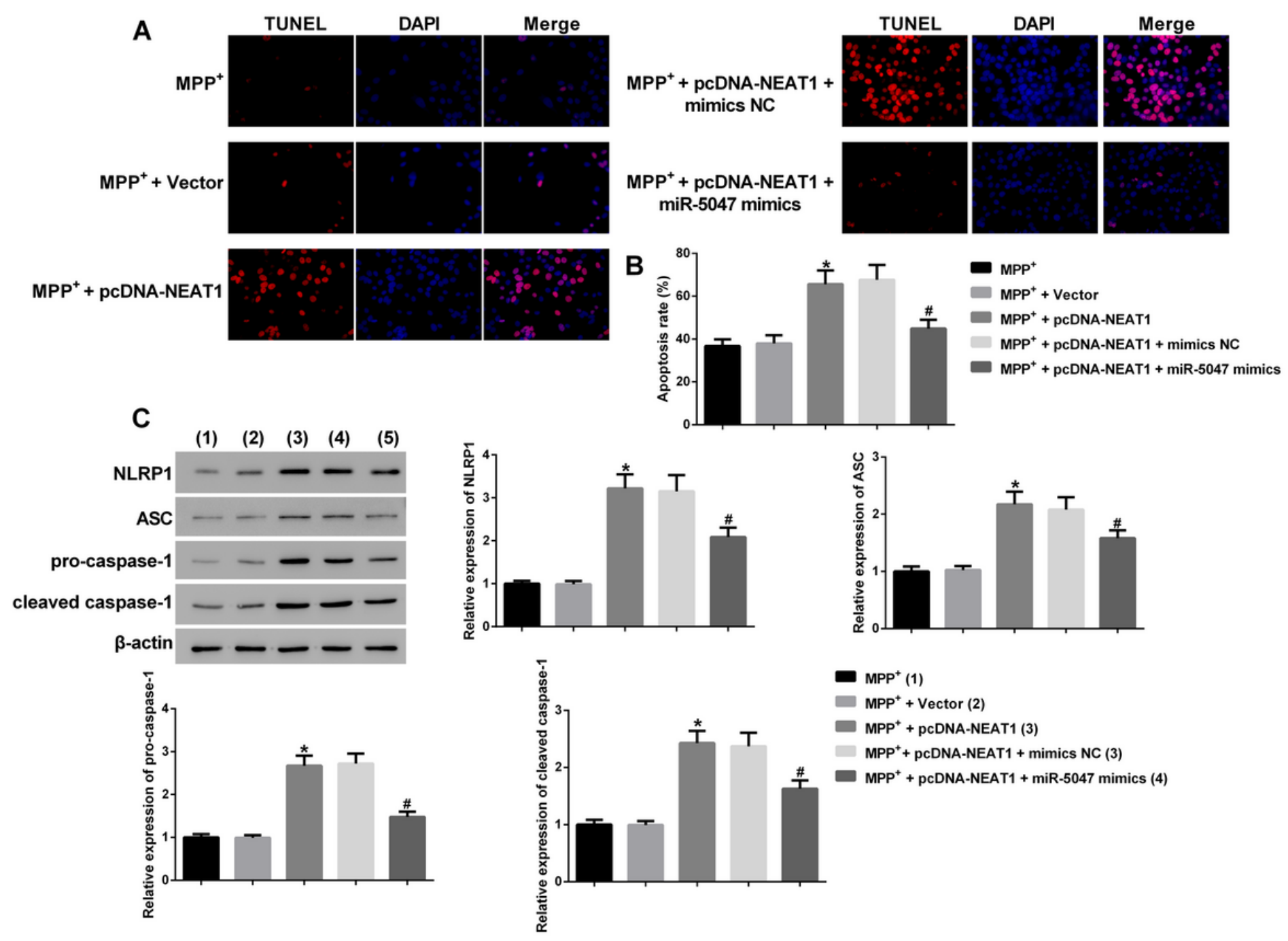

Figure 4

NEAT1 regulates SH-SY5Y cells pyroptosis through miR-5047. NEAT1 overexpression plasmids were cotransfected with miR-5047 mimics into SH-SY5Y cells. (A and B) The apoptotic rate of SH-SY5Y cells was detected through TUNEL staining. (C) The expression levels of NLRP1 inflammasomes-mediated pyroptosis-related proteins were measured by Western blot. ${ }^{\star} P<0.05 \mathrm{vs}$. MPP ${ }^{+}+$Vector group, and ${ }^{\#} P<$ 0.05 vs. $\mathrm{MPP}^{+}+$pcDNA-NEAT1 group. 
A
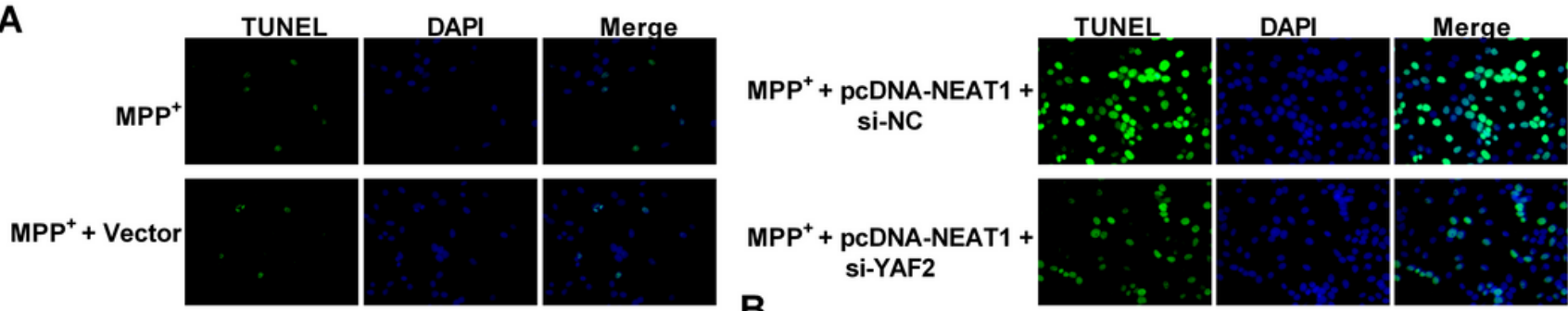

$\mathrm{MPP}^{+}+$pcDNA-NEAT1
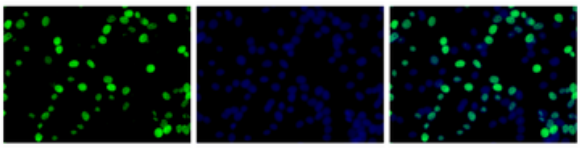

B
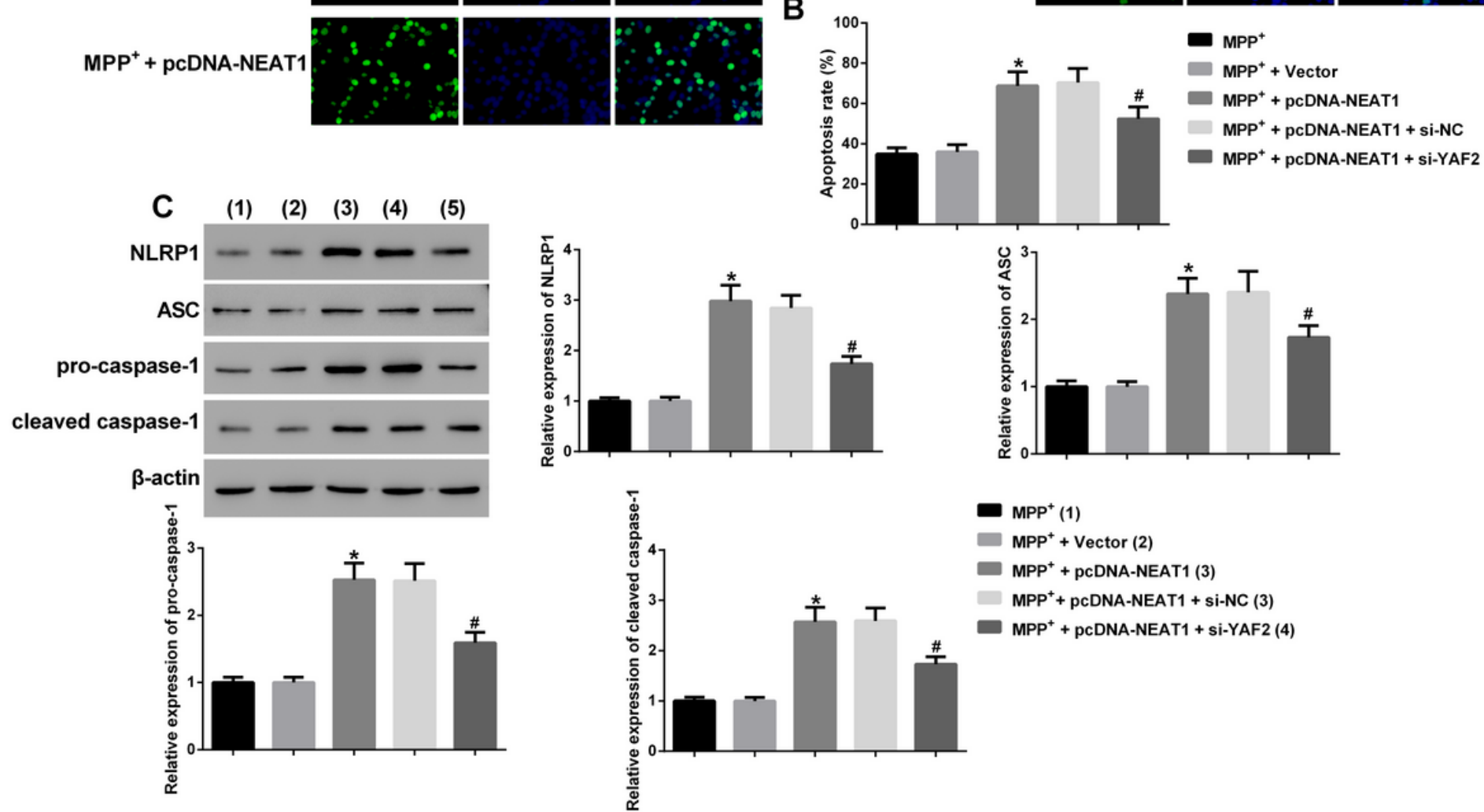

\section{Figure 5}

NEAT1 regulates SH-SY5Y cells pyroptosis through YAF2. NEAT1 overexpression plasmids were cotransfected with YAF2 siRNA into SH-SY5Y cells. (A and B) The apoptotic rate of SH-SY5Y cells was detected through TUNEL staining. (C) The expression levels of NLRP1 inflammasomes-mediated pyroptosis-related proteins were measured by Western blot. ${ }^{\star} P<0.05$ vs. $\mathrm{MPP}^{+}+$Vector group, and ${ }^{\#} P<$ 0.05 vs. MPP ${ }^{+}+$pcDNA-NEAT1 group.

\section{Supplementary Files}

This is a list of supplementary files associated with this preprint. Click to download.

- Supplementaryfigure1.tif

- Supplementaryfigure2.tif 